\title{
GPCRs of Diverse Physiologic and Pathologic Effects With \\ Fingerprints in COVID-19
}

Reza Nejat *,1, 2, Ahmad Shahir Sadr 3, 4, David J. Najafi 5, 6

${ }^{1}$ Former Assistant Prof., Department of Anesthesiology and Critical Care Medicine, Shahid Beheshti University of Medical Sciences, Tehran, Iran

${ }^{2}$ Former ICU Chief, Bazarganan Hospital, Tehran, Iran

${ }^{3}$ Bioinformatics Research Center, Cheragh Medical Institute and Hospital, Kabul, Afghanistan

${ }^{4}$ Department of Computer and Data Sciences, Faculty of Mathematical Sciences, Shahid Beheshti University, Tehran, Iran

${ }^{5}$ Assistant Clinical Professor, Shiley Eye Institute, UCSD, California

${ }^{6}$ Alliance Retinal Consultants, La Mesa California

*Corresponding Author Email: rezanejat@yahoo.com

Key words: ACE2; AT1R; Ang II; AT2R; angiotensin (1-7); $\beta$-arrestin; CFTR; COVID-19; ENaC; GPCR; MERSCoV; SARS-CoV2; SARS-CoV; G-protein

Abbreviation: ACE2: angiotensin converting enzyme 2; Ang II: angiotensin II; AT1R: angiotensin II type I receptor; AT2R: angiotensin II type II receptor; GAP: GTPase activating proteins; GPCR: G-protein-coupled receptors; GRK: GPCR-kinases; PKA: phosphokinase A; PKC: phosphokinase C; PLC: phospholipase C; RAS: Renin Angiotensin System;

\section{Abstract}

G-protein-coupled receptors (GPCR) belong to a large family of molecules eliciting different responses to a variety of signaling molecules. These receptors participate in various physiologic pathways such as metabolism, growth, immune responses, inflammation, vision, taste, olfaction, neurotransmission and even and pathologic responses including chronic inflammatory and vascular diseases. Receptors contributing to the biological responses of renin-angiotensin system (RAS) are members of GPCR family.

COVID-19-induced inflammatory cascade has been attributed to acute ACE2 downregulation and imbalance of proinflammatory ACE/AngII/AT1R and anti-inflammatory ACE2/angiotensin (17)/Mas axes in favor of the former. Some of the receptors contributing to activities of proteins in RAS including AT1R, AT2R and Mas receptors are members of GPCR family. It is notable that these receptors induce their effects both through $G$ protein and $\beta$-arrestin pathway; the former exerts temporary and the latter more sustained effects. In addition to the imbalance of GPCR responses contributing to RAS activities, it has been suggested that SARS-CoV2 pathogenesis might be attributed to the activation of GPCRs or modulating G-proteins involved in adenosine-CFTR regulation system and epithelial Na channel function. 
This article includes a minireview about the physiological functions of GPCRs and their contribution to COVID-19.

\section{Introduction:}

Cell-signaling, an adaptive strategy of cells in interaction with the environment, involves cellular chemical interaction to provoke appropriate responses to increase their survival. Cells release signaling molecules to the environment to bind to the receptors expressed in and on themselves or on neighboring or remote cells to initiate, integrate or coordinate their activities [1]. Receptors are of different families. G-protein-coupled receptors (GPCR), as a large family of proteins encoded in mammalian genome, contribute to many physiologic functions including vision, olfaction, taste, neurotransmission, metabolism, cell differentiation, immunity, inflammation, and prohormone signaling and if dysregulated, could lead to several pathologic processes including hypersensitivity to angiotensin II, inflammatory and vascular diseases [2-4]. These receptors share a common structure composed of a single polypeptide with an extracellular $\mathrm{N}$ terminus, an intracellular C-terminus and a hydrophobic seven-transmembrane domain (TM) connected by 3 intracellular and 3 extracellular loops [5]. GPCR family based on amino acid sequence and functional similarity are classified to six classes (A-F); vertebrates do not possess $D$ and E classes. Vertebrate GPCRs, according to GRAFS classification, are categorized into Glutamate (class C), Rhodopsin (class A), Adhesion, Frizzled and Secretin families $[4,6]$.

Upon binding with agonistic ligands, GPCRs adopt a proton-transport dependent conformational change and activate cytoplasmic heterotrimeric $G$ proteins ( $G \alpha / G \beta \gamma$ subunits) through dissociation of G $\alpha$ from $G \beta \gamma$ complex triggered by exchange of GTP for GDP in Ga subunit $[7,8]$. This process, depending on the subtype of $\mathrm{G} \alpha$ subunit, activates a second messenger including CAMP, inositol phosphates, $\mathrm{Ca}^{2+}$ and diacylglycerol which induces some intracellular pathways such as MAPK, PI3K-Akt and Ras and Rho GTPases [9, 10]. Based on the structure and function of $G \alpha$ subunit, $G$ proteins are divided into four subtypes: $G_{s}, G_{0} / i, G_{q / 11}$ and $G_{12 / 13}$. $G_{s}$ subtype contributes to activation adenyl cyclase, $\mathrm{G}_{\mathrm{o} / \mathrm{i}}$ inhibits adenyl cyclase and induces ion transport, $\mathrm{G}_{\mathrm{q} / 11}$ activates phospholipase $\mathrm{C}(\mathrm{PLC})$ and $\mathrm{G}_{12 / 13}$ is involved in $\mathrm{Na}^{+} / \mathrm{H}^{+}$exchange pathway [11].

It is of paramount importance that ligand-GPCR interaction is not a simple on/off switch. In fact, GPCR stimulation, depending on the energy transferred between ligand and G protein, is a complex phenomenon and may lead to multiple responses so that specific ligands may induce different pathways. Ligands may function as full, partial or inverse agonist and neutral antagonist [12]. Even GPCR signaling system has recently been uncovered to be capable of responding to different concentrations of the cognate ligand with distinct responses [13].

After propagation of the response, hydrolysis of GTP to GDP by the GTPase intrinsic activity of $\mathrm{G} \alpha$ subunit ends $\mathrm{G}$ protein signaling transduction. GTPase activating proteins (GAPs) and regulators of $G$ protein signaling (RGS) accelerate this reaction. This results in recreation of $\mathrm{G} \alpha / \mathrm{G} \beta \gamma$ complex for the next signal transduction [13]. Alternatively, after activation of GPCRs, phosphorylation of Serine and Threonine residues on C-terminal end or third intracellular loop of 
the receptors by GPCR-kinases (GRK) creates a binding site for $\beta$-arrestins. Binding with $\beta$ arrestins shuts down G-dependent signaling cascades through desensitization of the receptor to the next ligand or internalization of the receptor and promotion of multiple G-independent pathways by inducing different protein kinase dependent pathways [14-16].

Intriguingly, GPCRs can also be found within the cells residing on membranous organelles such as endoplasmic reticulum, Golgi apparatus and endosomes with the ability to translocate to the nucleus. After internalization, AT1R, apelin and bradykinin2 receptors as well as receptors for chemokines may use canonical nuclear localization signals (NLSs) recognized by karyopherin superfamily for nuclear import $[17,18]$.

\section{COVID-19 and GPCRs}

SARS-CoV2, one of the members of $\beta$-family of coronaviruses and the cause of COVID-19 has resulted in a pandemic with huge social economical and healthcare burden [19]. The resulting insult leads to acute inflammation in the lungs similar to the pathology seen in acute respiratory distress syndrome. It is worth mentioning that SARS-CoV2 like SARS-CoV evades the immune system $[20,21]$. Accordingly, failure to activate a well-orchestrated effective innate immunity leads to eruption of an abortive adaptive immunity with the inability to resolve the pathology and clearance of infected cells. This results in sequential uninterrupted production of inflammatory mediators and eventually cytokine storm [22, 23]. Based on a great amount of data, the elicited profound inflammatory responses in COVID-19 has been attributed to the imbalance in RAS [24]. This virus on entry the host cell uses and downregulates its receptor, ACE2, a transmembrane carboxy-monopeptidase of renin-angiotensin system (RAS) which converts Ang II to angiotensin (1-7) [25, 26]. Acute downregulation of ACE2, as may occur in showering of huge number of SARS-CoV2 virions to the lower airways, results in acute imbalance of the two major arms of local RAS, ACE/Ang II/AT1R and ACE2/ang (1-7)/Mas, in favor of the former [27]. ACE/Ang II/AT1R elicits pro-inflammatory, pro-thrombotic, pro-apoptotic responses while ACE2/ang (17)/Mas predominantly presents anti-inflammatory, anti-thrombotic and anti-apoptotic effects [28]. It should be noted that AT1R, AT2R and Mas receptors belong to GPCRs [29, 30]. While sustained Angll activation of AT1R induces inflammatory responses through G-proteins, angiotensin(1-7) promotes anti-inflammatory effects both via Mas/GPCR receptors and AT1R/GPCR mediated $\beta$-arrestin pathway [31]. This imbalance may ultimately result in severe inflammatory responses and multiple organ failure with utmost similarity to macrophage activation syndrome or other kinds of cytokine storms [27, 32].

SARS-CoV2 has been suggested to induce lung edema through cystic fibrosis transmembrane conductance regulator (CFTR) system and epithelial $\mathrm{Na}^{+}$channel (ENaC) function [33, 34]. CFTR predominantly regulated by CAMP/PKA, the second messenger of GPCR system, inhibits ENaC function, yet to be elucidated meticulously $[35,36]$. ENaC as a member of voltage-independent $\mathrm{Na}^{+}$-selective ENaC/DEG ion channels is involved in airway and alveolar space fluid clearance by 
epithelial and alveolar cells of the lungs [37, 38]. Alternatively, SARS-CoV and some viruses infecting the lungs including influenza virus were shown to decrease the activity of ENaC through activation of phosphokinase C (PKC) [39, 40]. PKC, upregulated by extracellular purinergic receptors which are themselves of or intricately interact with GPCR family, may exert negative influence on ENaC cell membrane trafficking and expression [41, 42]. In addition, GPCR affects ENaC activity through $\mathrm{PI}(4,5) \mathrm{P}[43]$.

Furthermore, complement 5a receptor1 (C5aR1), a member of GPCR family, has recently been proposed to be involved in COVID-19 pathogenesis [44]. GPCR4, which regulates vascular permeability and leukocyte recruitment, has also been hypothesized to play a part in SARS-CoV2 infection [45].

Conclusion and Future Perspectives: GPCRs, as a large family of molecules in cell signaling systems, contribute to a huge number of physiologic and pathologic responses. The effects of these receptors can be traced in a different array of infections. As to the complex pathogenesis of SARS-CoV2 infection which requires the contribution of GPCRs and the sustained responses these receptors may exert in intracellular scales, they might be a good target to produce drugs in order to restrict this infection and improve the long-term consequences of the disease.

Acknowledgement: The authors would like to extend their sincere gratitude to all healthcare personnel around the world who sacrifice their lives to take care of COVID-19 patients.

Funding: This research received no external funding.

Conflicts of Interest: The authors declare no conflict of interest.

\section{References:}

1. Cooper, G.M., Cell Signaling, in The Cell-A Molecular Approach. 2019, Oxford University Press U.S.A. p. 565.

2. Quitterer, U. and S. AbdAlla, Discovery of pathologic GPCR aggregation. Frontiers in medicine, 2019. 6: p. 9.

3. Zalewska, M., M. Siara, and W. Sajewicz, G protein-coupled receptors: abnormalities in signal transmission, disease states and pharmacotherapy. Acta Pol Pharm, 2014. 71(2): p. 229-243.

4. Hu, G.-M., T.-L. Mai, and C.-M. Chen, Visualizing the GPCR network: classification and evolution. Scientific reports, 2017. 7(1): p. 1-15.

5. Alexander, S.P., et al., The Concise Guide to PHARMACOLOGY 2019/20: G protein-coupled receptors. British journal of pharmacology, 2019. 176: p. S21-S141.

6. Basith, S., et al., Exploring G protein-coupled receptors (GPCRs) ligand space via cheminformatics approaches: impact on rational drug design. Frontiers in pharmacology, 2018. 9: p. 128.

7. Rajagopal, S., K. Rajagopal, and R.J. Lefkowitz, Teaching old receptors new tricks: biasing seventransmembrane receptors. Nature reviews Drug discovery, 2010. 9(5): p. 373-386.

8. Zhang, X.C., et al., Proton transfer-mediated GPCR activation. Protein \& cell, 2015. 6(1): p. 12-17.

9. Walsh, C.T., D. Stupack, and J.H. Brown, G Protein-Coupled Receptors Go Extracellular: RhoA Integrates the Integrins. Molecular interventions, 2008. 8(4): p. 165. 
10. Otto, J.C., et al., Alterations in an inositol phosphate code through synergistic activation of a $G$ protein and inositol phosphate kinases. Proceedings of the National Academy of Sciences, 2007. 104(40): p. 15653-15658.

11. $\mathrm{Li}, \mathrm{Z}$., et al., Classification of $\mathrm{G}$ proteins and prediction of GPCRs-G proteins coupling specificity using continuous wavelet transform and information theory. Amino acids, 2012. 43(2): p. 793-804.

12. Rosenbaum, D.M., S.G. Rasmussen, and B.K. Kobilka, The structure and function of G-proteincoupled receptors. Nature, 2009. 459(7245): p. 356-363.

13. Keshelava, A., et al., High capacity in $G$ protein-coupled receptor signaling. Nature communications, 2018. 9(1): p. 1-8.

14. Tilley, D.G., G protein-dependent and G protein-independent signaling pathways and their impact on cardiac function. Circulation research, 2011. 109(2): p. 217-230.

15. Peterson, Y.K. and L.M. Luttrell, The diverse roles of arrestin scaffolds in $G$ protein-coupled receptor signaling. Pharmacological reviews, 2017. 69(3): p. 256-297.

16. Turu, G., A. Balla, and L. Hunyady, The role of B-arrestin proteins in organization of signaling and regulation of the AT1 angiotensin receptor. Frontiers in endocrinology, 2019. 10: p. 519.

17. Jong, Y.J.I., S.K. Harmon, and K.L. O'Malley, GPCR signalling from within the cell. British journal of pharmacology, 2018. 175(21): p. 4026-4035.

18. Eichel, K. and M. von Zastrow, Subcellular organization of GPCR signaling. Trends in Pharmacological Sciences, 2018. 39(2): p. 200-208.

19. Andronico, A., et al., Evaluating the impact of curfews and other measures on SARS-CoV-2 transmission in French Guiana. Nature communications, 2021. 12(1): p. 1-8.

20. Molaei, S., et al., The immune response and immune evasion characteristics in SARS-CoV, MERSCoV, and SARS-CoV-2: Vaccine design strategies. International immunopharmacology, 2020: p. 107051.

21. Nejat, R. and A.S. Sadr, SARS virus papain-like protease: a mysterious weapon. Journal of Biostatistics and Epidemiology, 2019. 5(4): p. 288-295.

22. Tufet, M., T cells calm the storm. Nature Reviews Immunology, 2007. 7(11): p. 834-835.

23. Henderson, L.A., et al., On the alert for cytokine storm: immunopathology in COVID-19. Arthritis \& Rheumatology, 2020. 72(7): p. 1059-1063.

24. Nejat, R. and A.S. Sadr, Are losartan and imatinib effective against SARS-CoV2 pathogenesis? A pathophysiologic-based in silico study. In silico pharmacology, 2021. 9(1): p. 1-22.

25. Hamming, I., et al., The emerging role of ACE2 in physiology and disease. The Journal of Pathology: A Journal of the Pathological Society of Great Britain and Ireland, 2007. 212(1): p. 1-11.

26. Shang, J., et al., Cell entry mechanisms of SARS-CoV-2. Proceedings of the National Academy of Sciences, 2020. 117(21): p. 11727-11734.

27. Banu, N., et al., Protective role of ACE2 and its downregulation in SARS-CoV-2 infection leading to Macrophage Activation Syndrome: Therapeutic implications. Life sciences, 2020: p. 117905.

28. Reddy Gaddam, R., S. Chambers, and M. Bhatia, ACE and ACE2 in inflammation: a tale of two enzymes. Inflammation \& Allergy-Drug Targets (Formerly Current Drug Targets-Inflammation \& Allergy)(Discontinued), 2014. 13(4): p. 224-234.

29. Magnani, F., et al., Electronic sculpting of ligand-GPCR subtype selectivity: the case of angiotensin II. ACS chemical biology, 2014. 9(7): p. 1420-1425.

30. Santos, R.A., et al., Angiotensin-(1-7) is an endogenous ligand for the G protein-coupled receptor Mas. Proceedings of the National Academy of Sciences, 2003. 100(14): p. 8258-8263.

31. Manglik, A., et al., 6-Arrestin-Biased Angiotensin II Receptor Agonists for COVID-19. Circulation, 2020. 142(4): p. 318-320.

32. Iwasaki, M., et al., Inflammation triggered by sars-cov-2 and ace2 augment drives multiple organ failure of severe covid-19: Molecular mechanisms and implications. Inflammation, 2020: p. 1-22. 
33. Abdel Hameid, R., et al., SARS-CoV-2 may hijack GPCR signaling pathways to dysregulate lung ion and fluid transport. American Journal of Physiology-Lung Cellular and Molecular Physiology, 2021. 320(3): p. L430-L435.

34. $\mathrm{Yu}$, L., et al., The inhibitory effect of $G B Y$ and $G B$ isoform specificity on ENaC activity. American Journal of Physiology-Renal Physiology, 2013. 305(9): p. F1365-F1373.

35. Saint-Criq, V. and M.A. Gray, Role of CFTR in epithelial physiology. Cellular and Molecular Life Sciences, 2017. 74(1): p. 93-115.

36. Berdiev, B.K., Y.J. Qadri, and D.J. Benos, Assessment of the CFTR and ENaC association. Molecular BioSystems, 2009. 5(2): p. 123-127.

37. Noreng, S., et al., Structure of the human epithelial sodium channel by cryo-electron microscopy. Elife, 2018. 7: p. e39340.

38. Planès, C., et al., ENaC-mediated alveolar fluid clearance and lung fluid balance depend on the channel-activating protease 1. EMBO molecular medicine, 2010. 2(1): p. 26-37.

39. Ji, H.-L., et al., SARS-COV proteins decrease levels and activity of human ENaC via activation of distinct PKC isoforms. American Journal of Physiology-Lung Cellular and Molecular Physiology, 2009. 296(3): p. L372-L383.

40. Chen, X.-J., et al., Influenza virus inhibits ENaC and lung fluid clearance. American Journal of Physiology-Lung Cellular and Molecular Physiology, 2004. 287(2): p. L366-L373.

41. Bhalla, V. and K.R. Hallows, Mechanisms of ENaC regulation and clinical implications. Journal of the American Society of Nephrology, 2008. 19(10): p. 1845-1854.

42. Bilbao, P.S., S. Katz, and R. Boland, Interaction of purinergic receptors with GPCRs, ion channels, tyrosine kinase and steroid hormone receptors orchestrates cell function. Purinergic signalling, 2012. 8(1): p. 91-103.

43. Pochynyuk, O., et al., Binding and direct activation of the epithelial Na+ channel (ENaC) by phosphatidylinositides. The Journal of physiology, 2007. 580(2): p. 365-372.

44. Carvelli, J., et al., Association of COVID-19 inflammation with activation of the C5a-C5aR1 axis. Nature, 2020: p. 1-5.

45. Yang, L.V., et al., Can GPR4 Be a Potential Therapeutic Target for COVID-19? Frontiers in medicine, 2021. 7: p. 1150. 